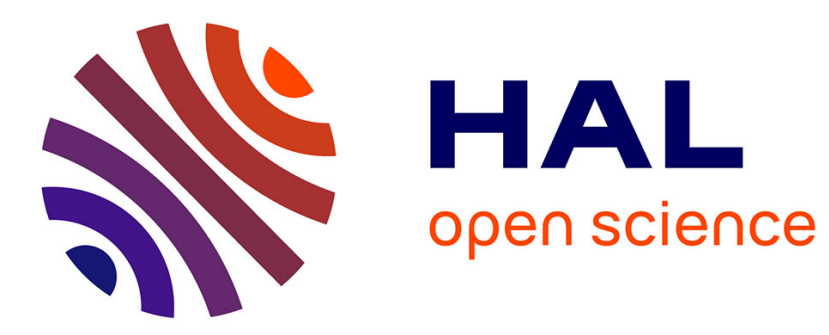

\title{
Permeability changes in coal seams: the role of anisotropy
}

\author{
L.L. Wang, Matthieu Vandamme, Jean-Michel Pereira, Patrick Dangla, \\ Nicolas Espinoza
}

\section{- To cite this version:}

L.L. Wang, Matthieu Vandamme, Jean-Michel Pereira, Patrick Dangla, Nicolas Espinoza. Permeability changes in coal seams: the role of anisotropy. International Journal of Coal Geology, 2018, 199, pp.52-64. 10.1016/j.coal.2018.09.014 . hal-02125805

\section{HAL Id: hal-02125805 \\ https://hal-enpc.archives-ouvertes.fr/hal-02125805}

Submitted on 10 May 2019

HAL is a multi-disciplinary open access archive for the deposit and dissemination of scientific research documents, whether they are published or not. The documents may come from teaching and research institutions in France or abroad, or from public or private research centers.
L'archive ouverte pluridisciplinaire HAL, est destinée au dépôt et à la diffusion de documents scientifiques de niveau recherche, publiés ou non, émanant des établissements d'enseignement et de recherche français ou étrangers, des laboratoires publics ou privés. 


\title{
Permeability changes in coal seams: the role of anisotropy
}

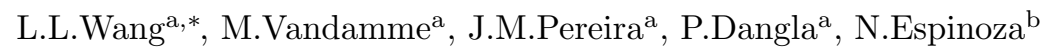 \\ ${ }^{a}$ Laboratoire Navier, Université Paris-Est, École des Ponts ParisTech, \\ 77455 Marne-la-Vallée Cedex, France \\ ${ }^{b}$ Department of Petroleum and Geosystems Engineering, The University of Texas at Austin, United States
}

\begin{abstract}
The permeability of coal seams is pore pressure-dependent. A number of analytical models have been proposed to investigate this problem, but many disregard a crucial factor: the anisotropy of coal. This paper is devoted to investigating the role of anisotropy in modeling the change of horizontal permeability with pressure. Analysis is conducted using a fully anisotropic model that incorporates both the anisotropies of mechanical properties and of the permeability dependence on stresses. Analytical expressions of the pressure-permeability relationship are derived in oedometric and isochoric geomechanical conditions, and validations are conducted against both laboratory and field data. Then, the roles of the anisotropy of stiffness and of permeability dependence on stresses in the permeability-change model are explored. We demonstrate that the mechanical anisotropy can be simplified to an isotropic model without introducing significant errors in prediction of pressure-permeability relationship, while neglecting the anisotropy of permeability dependence on stresses leads to considerable errors. When both anisotropy sources are disregarded, the pressure-permeability curve can be exactly reproduced by a totally isotropic material in both oedometric and isotropic conditions. However, the material properties (e.g., bulk modulus) are skewed; moreover, such an equivalent material might lead to significant errors in other geomechanical conditions. Finally, the permeability change is investigated at the reservoir scale, and the reservoir simulation results confirm the conclusions obtained from the analytical analysis.
\end{abstract}

Keywords: Coalbed methane, permeability, anisotropy, analytical modeling, reservoir simulation

\section{Introduction}

Coalbed methane $(\mathrm{CBM})$ is an important source of energy in the United States, Canada, Australia, and China among other countries. Different from other geomaterials encountered in natural gas recovery,

\footnotetext{
*Corresponding author, now at China University of Petroleum, Beijing

Email addresses: linlin.wang@cup.edu.cn (L.L.Wang), matthieu.vandamme@enpc.fr (M.Vandamme), jeanmichel.pereira@enpc.fr (J.M.Pereira), patrick.dangla@iffstar.fr (P.Dangla), espinoza@austin.utexas.edu (N.Espinoza) 
coalbeds are conferred unique poromechanical properties by their two-scale porosity systems: macro-porosity and micro-porosity (Espinoza et al., 2014; Nikoosokhan et al., 2014). The macro-pores in coalbeds are commonly constituted by cleats, that are, natural fractures developed during diagenetic processes (Laubach et al., 1998). The cleats act as the major channel for methane to flow and thus govern the permeability of fractured coal seams. Between cleats, one finds a microporous organic continuum, routinely called as coal matrix. Methane is stored inside the micro-pores of coal matrix. In the micro-pores (typically sized in the order of $10^{-9}$ to $10^{-8} \mathrm{~m}$ ), all fluid molecules interact with the atoms of solid matrix; they are therefore not in their bulk state as in the macro-pores but are in adsorbed state (Vandamme et al., 2010; Brochard et al., 2012). The adsorbed state of methane in micro-pores governs the adsorption/desorption phenomena and subsequent swelling/shrinking of coal matrix (Pan and Connell, 2007; Day et al., 2008).

The permeability of fractured CBM reservoirs changes with depletion during production, and this process plays an important role during production and enhanced recovery operations. The permeability change with depletion (i.e., decrease of pore pressure) mainly stems from two mechanisms with opposing effects. The first mechanism involves the mechanical deformation due to pressure changes: with decreasing pressure, an increase in effective stress leads to compression of coal and reduction in cleat porosity, so the permeability decreases. The second mechanism is desorption-induced shrinkage of the coal matrix with depletion, resulting in an increase in cleat aperture and thus a rise in permeability.

Several analytical models of pressure-dependent permeability have been proposed such as P-M model (Palmer and Mansoori, 1998; Palmer et al., 2007), S-D model (Shi and Durucan, 2004), C-B model (Cui and Bustin, 2005; Cui et al., 2007). A detailed review of these models can be found in Shi and Durucan (2004); Connell (2009); Palmer (2009). These models can be broadly classified into two categories: strain-based and stress-based. The strain-based models relate the permeability $(k)$ to the cleat porosity $\left(\phi_{c}\right)$ using the bundled-matchstick conceptual model (Seidle et al., 1992), e.g.: $k / k_{0}=\left(\phi_{c} / \phi_{c 0}\right)^{3}$, where the subscript 0 refers to a reference state. For the stress-based models, the permeability is related to effective stress, $\sigma^{\prime}$, e.g.: $k / k_{0}=e^{-3 \alpha\left(\sigma^{\prime}-\sigma_{0}^{\prime}\right)}$, where $\alpha$ is cleat (volume) compressibility. These models are supported by a wide variety of experimental measurements and have been useful for history-matching various CBM plays. In modeling the permeability evolution, the anisotropy of coal is a crucial influencing factor. This factor has been investigated (Day et al., 2008; Wang et al., 2009; Pan and Connell, 2011; Wang et al., 2013, 2014) but needs to be further explored.

Considering the stress-based models, anisotropy exists in two aspects: the mechanical behaviour (to compute the effective stress) and the permeability dependence on stresses (related to cleat compressibil- 

of the dual-porosity system, the mechanical anisotropy of coal seams is controlled by two opposite effects. Regarding the coal matrix, the lamination and preferred orientation of the macerals during sedimentation, compaction and diagenesis processes contribute to an intrinsic anisotropy of the coal matrix: the horizontal Young's modulus is greater than the vertical one, $E / E_{3}>1$ (the axis 3 is vertical). Thus, this intrinsic anisotropy increases with the degree of maturation, as demonstrated by Morcote et al. (2010). Regarding the cleats, they are prone to be sub-vertically oriented and thus counteract the effect of bedding. In theory, the compressibility of vertical cleats can lead $E / E_{3}$ at the seam scale to be less than 1 . The cleats are sensitive to the confining stress: sealing the vertical cleats leads the anisotropy to be more and more dominated by the fabric and so causes $E / E_{3}$ to increase. Combining the two opposite effects, the anisotropy of elastic properties of coal seams is variable, depending on the degree of maturation, on the confining stress, and on the size of tested samples (number of cleats). For instance, the ultrasonic velocity measurements by Morcote et al. (2010) revealed that horizontal P-wave velocities are greater than vertical velocities for three types of coal. Espinoza et al. (2014); Hol and Spiers (2012) found $E$ and $E_{3}$ are comparable. However, Pone et al. (2010) showed that, for a coal sample exposed to 6.9 MPa hydrostatic stress, the $y$-axis strain is greater than the $z$-axis strain ( $z$-axis is the vertical direction). Beside the mechanical anisotropy, how permeability depends on effective stresses is also anisotropic. As shown by Espinoza et al. (2014), variation of horizontal effective stress leads to more change in horizontal permeability compared to variation of vertical effective stress with the same magnitude, indicating the anisotropy of the cleat compressibility $\alpha$.

This paper is devoted to investigating the role of anisotropy in the permeability changes in coal seams. It should be noted that the permeability of coal seams is anisotropic: the horizontal permeability is different from the vertical one. However, the present work focuses on the horizontal permeability only. This is because CBM formations are commonly thin, and hence mostly horizontal flow is involved in the production of methane. Therefore, for what concerns CBM processes, the horizontal permeability predominates, and the role of the vertical permeability is secondary. In this sense, the term "permeability" used in the present work specifically refers to the horizontal permeability. In summary, the paper focuses on the role of anisotropies (i.e., anisotropy of the mechanical properties and anisotropy of the dependence of permeability on stresses) in the change of the horizontal permeability, not on the anisotropy of permeability itself. The paper is composed of four parts. After the introduction, the theoretical background used for the analysis is shortly recalled, including a transverse isotropic poromechanical model and a permeability law that depends in a transversely isotropic manner on effective stresses. Then, the role of anisotropy is investigated by analytical 
modeling at the scale of a representative element volume (REV) as well as numerical modeling at the scale of a CBM reservoir.

\section{Theory}

\subsection{Transverse isotropic poromechanical model}

The poromechanical model used in this work is a double porosity transverse isotropic model developed by Espinoza et al. (2014). In brief, the model for coal seams is based on poromechanical equations that explicitly take into account the effect of adsorption on the mechanical behavior of a microporous medium (Brochard et al., 2012). As discussed previously, the double porosity system of coal seams is composed of 1) the cleat macro-porosity where the fluid is in bulk state, and 2) the micro-porosity within the coal matrix where the fluid is in adsorbed state, and where adsorptive-mechanical couplings (e.g., swelling or shrinking) originate. The contribution of cleat compressibility to the poromechanical behaviour of coal seams is modelled using conventional anisotropic poroelasticity (Cheng, 1997); the effect of adsorption-induced phenomena in the coal matrix is modelled using the theory of generalized poromechanics for microporous media developed by Brochard et al. (2012) and Espinoza et al. (2013). The model equations are established from a thermodynamical formulation on the basis of energy conservation. Details about the model derivation can be found in Espinoza et al. (2014), and a more detailed formulation for isotropic solids can be found in Nikoosokhan et al. (2014). We merely recall the model equations (the symmetry is around axis 3):

$$
\left\{\begin{aligned}
\Delta \sigma_{11} & =C_{11} \Delta \varepsilon_{11}+C_{12} \Delta \varepsilon_{22}+C_{13} \Delta \varepsilon_{33}-b_{1} \Delta P_{c}-\left(1-b_{1}\right) \Delta S^{a}\left(P_{m}\right) \\
\Delta \sigma_{22} & =C_{12} \Delta \varepsilon_{11}+C_{11} \Delta \varepsilon_{22}+C_{13} \Delta \varepsilon_{33}-b_{1} \Delta P_{c}-\left(1-b_{1}\right) \Delta S^{a}\left(P_{m}\right) \\
\Delta \sigma_{33} & =C_{13} \Delta \varepsilon_{11}+C_{13} \Delta \varepsilon_{22}+C_{33} \Delta \varepsilon_{33}-b_{3} \Delta P_{c}-\left(1-b_{3}\right) \Delta S^{a}\left(P_{m}\right) \\
\Delta \sigma_{23} & =2 C_{44} \Delta \varepsilon_{23} \\
\Delta \sigma_{31} & =2 C_{44} \Delta \varepsilon_{31} \\
\Delta \sigma_{12} & =\left(C_{11}-C_{12}\right) \Delta \varepsilon_{12} \\
\Delta \phi_{c} & =b_{1}\left(\Delta \varepsilon_{11}+\Delta \varepsilon_{22}\right)+b_{3} \Delta \varepsilon_{33}+\left[\Delta P_{c}-\Delta S^{a}\left(P_{m}\right)\right] / N \\
n_{T} & =\left(1-\phi_{c 0}\right) n_{m}\left(P_{m}, \varepsilon_{m}\right)+\rho_{b} \phi_{c}
\end{aligned}\right.
$$

The first seven equations are the conventional anisotropic poroelasticity added with a term $S_{a} . \sigma_{i j}$ and $\varepsilon_{i j}$ are total stress and strain tensors defined at the coal seam scale; $P_{c}$ is the pressure of fluid in the cleat. $C_{11}, C_{12}, C_{13}, C_{33}, C_{44}$ are the five independent stiffness coefficients; $b_{1}$ and $b_{3}$ are cleat-induced 
Biot coefficients; $N$ is Biot modulus; $\phi_{c}$ is cleat porosity. The term $S_{a}$ quantifies the stress needed to keep the coal matrix at zero volumetric strain during adsorption (see the first three equations), so it is termed adsorption stress. The last eighth equation describes the total amount of fluid in per unit volume of coal seam, $n_{T}$, as the sum of two terms: 1) the amount of fluid adsorbed in the matrix, $n_{m}$, and 2) the amount of fluid in the cleats, which is equal to the cleat porosity, $\phi_{c}$, multiplied by the bulk fluid molar density, $\rho_{b}$. The coal matrix strain $\varepsilon_{m}$ is related to the volumetric strain at the coal seam REV scale $\varepsilon=\varepsilon_{11}+\varepsilon_{22}+\varepsilon_{33}$ through the following relation (Coussy, 2010):

$$
\varepsilon_{m}=\frac{\varepsilon-\left(\phi_{c}-\phi_{c 0}\right)}{1-\phi_{c 0}}
$$

where $\phi_{c 0}$ is the reference porosity of an undeformed fractured coal. The adsorbed fluid in the coal matrix, $n_{m}$, depends both on fluid pressure, $P_{m}$, and on volumetric strain, $\varepsilon_{m}$. Since strains of the coal matrix are small, the amount $n_{m}$ of adsorbed fluid can be approximated by its first-order expression with respect to $\varepsilon_{m}$ (Brochard et al., 2012):

$$
n_{m}\left(P_{m}, \varepsilon_{m}\right)=n_{m 0}\left(P_{m}\right)\left[1+c\left(P_{m}\right) \varepsilon_{m}\right]
$$

where $n_{m 0}$ is the adsorbed quantity at zero matrix strain. The adsorption isotherm is written here in form of Langmuir equation $n_{m 0}=n_{0}^{\max } P_{m} /\left(P_{L}+P_{m}\right)$, where $n_{0}^{\max }$ and $P_{L}$ are two constants. The term $c\left(P_{m}\right)$ is a coefficient that quantifies the effect of strain on adsorption capacity. This coupling coefficient is positive for fluids that make coal swell.

Using Equation (3), the adsorption stress can be expressed as:

$$
S^{a}\left(P_{m}\right)=\int_{0}^{P_{m}} n_{m 0}\left(P_{m}\right) c\left(P_{m}\right) V_{b}\left(P_{m}\right) d P_{m}
$$

where $V_{b}$ is the molar volume of the fluid in bulk conditions as a function of temperature and pressure. This term can be thought as an analog of thermal stress, but for adsorption (Espinoza et al., 2014). The $S^{a}-P_{m}$ relationship for methane is shown in Figure 1a.

The Biot coefficients $\left(b_{1}\right.$ and $\left.b_{3}\right)$ and Biot modulus $(N)$ are related to the coal matrix bulk modulus 


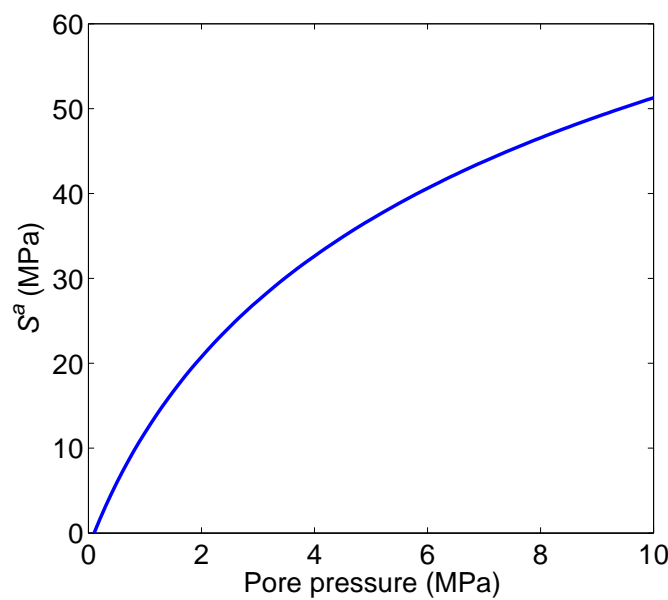

(a)

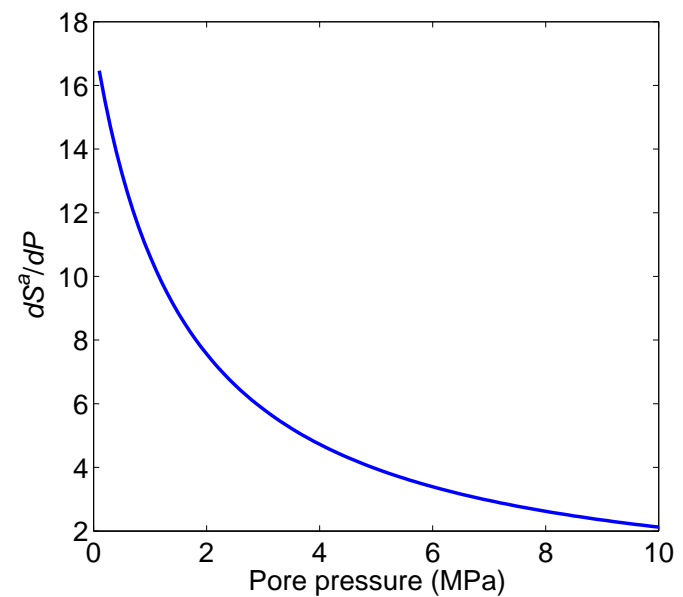

(b)

Figure 1: Adsorption stress as function of pore pressure (a) and its derivative (b).

$\left(K_{m}\right)$ as follows (Coussy, 2010),

$$
\left\{\begin{array}{l}
b_{1}=1-\frac{C_{11}+C_{12}+C_{13}}{3 K_{m}} \\
b_{3}=1-\frac{2 C_{13}+C_{33}}{3 K_{m}} \\
\frac{1}{N}=\frac{\left(2 b_{1}+b_{3}\right) / 3-\phi_{c 0}}{3 K_{m}}
\end{array}\right.
$$

According to Gibbs-Duhem equation, the pressure of fluid is related to the chemical potential as $d U=$ $V_{b}(P) d P$ for isothermal conditions. In this sense, the term $P_{m}$ is the pressure of the bulk fluid at the same chemical potential as the adsorbed phases in the coal matrix, termed thermodynamic pressure. In the following, the pressure of the fluid in the cleats and the thermodynamic pressure of the fluid in the coal matrix are assumed to be equal, implying fluid in the cleats is in thermodynamic equilibrium with fluid in the coal matrix at all times. Accordingly, the two terms $P_{c}$ and $P_{m}$ are presented by one term $P$, termed pore pressure. It is true that a pressure gradient can exist between the cleat and the coal matrix, especially in transient states (Peng et al., 2014). Hence, the assumption of local homogeneity of pore pressure is a limit to the applicability of the model to real cases.

The anisotropy of coal swelling (Day et al., 2008; Wang et al., 2009; Pan and Connell, 2011) is considered in the present work as a direct consequence of the anisotropy of the stiffness coefficients of the dry material. Indeed, Espinoza et al. (2013) showed that swelling anisotropy comes mostly from the anisotropy of the stiffness coefficients, and not so much from the anisotropy of the adsorption stress itself. Therefore, in the present work, we considered an isotropic adsorption stress; nevertheless, combined with anisotropic stiffness 
coefficients, this isotropic adsorption stress induces an anisotropic swelling.

\subsection{Permeability model}

As mentioned previously, the permeability-change models are commonly established by two means, strainbased and stress-based. We adopt here a stress-based model that correlates the logarithm of horizontal permeability to the horizontal and vertical Terzaghi's effective stresses, $\sigma$, such that,

$$
k=k_{0} \exp \left[2 \alpha_{h}\left(\Delta \sigma_{h}+\Delta P\right)+\alpha_{v}\left(\Delta \sigma_{v}+\Delta P\right)\right]
$$

where $k_{0}$ is the reference horizontal permeability at a reference state. This model independently incorporates the effects of the horizontal effective stress and of the vertical effective stress through introducing two cleat compressibility coefficients $\alpha_{h}$ and $\alpha_{v}$. This is different from the common used models that employed the mean effective stress or the horizontal effective stress (Somerton et al., 1975; Shi and Durucan, 2004; Cui et al., 2007).

\subsection{Reference material}

The reference coal for the analysis comes from Forzando mine in South Africa, at depth of $500 \mathrm{~m}$. Several cores were drilled in the horizontal and vertical directions. To calibrate the parameters of the adsorptive poromechanical model and of the permeability law in Espinoza et al. (2014), experiments were performed using a specifically designed triaxial cell that is able to 1) apply independently axial and radial stresses, 2) measure core axial and radial deformations, 3) measure gas uptake by the coal specimen, and 4) measure core permeability. The four elastic constants of dry coal specimens $\left(E, E_{3}, \nu, \nu_{3}\right)$ were measured from the stressstrain data on cores (drilled horizontally and vertically) under anisotropic loading in drained conditions. The coal matrix bulk modulus $\left(K_{m}\right)$ was chosen as the bulk modulus of the coal core for the highest applied confining stress $30 \mathrm{MPa}$. Cleat-induced Biot coefficients and Biot modulus $\left(b_{1}, b_{3}, N\right)$ were then obtained from Equation (5). The mean initial macroporosity $\left(\phi_{c 0}\right)$ was estimated subtracting the microporosity of coal matrix from the helium (total) porosity. The adsorptive properties $\left(n_{0}^{\max }, P_{L}, c\right)$ were obtained from simultaneous fitting of 1) the coal matrix total sorption data, 2) the total uptake measured in the triaxial experiments, 3) the change of strains upon injection, and 4) the measured swelling slopes upon predominant adsorption regime. With regards to the permeability law, the cleat permeabilities at different horizontal and vertical Terzaghi's effective stresses were measured at constant flow rate regimes. Then the values of fracture compressibility parameters $\left(\alpha_{h}, \alpha_{v}, k_{0}\right)$ were calculated. 
Table 1: Model parameters for the reference material.

\begin{tabular}{cc|cc|cc}
\hline \multicolumn{2}{c|}{ Core scale } & \multicolumn{2}{c|}{ Coal matrix } & \multicolumn{2}{c}{ Permeability } \\
\hline$E$ & $4082 \mathrm{MPa}$ & $K_{m}$ & $5000 \mathrm{MPa}$ & $k_{0}(10 \mathrm{MPa})$ & $0.0029 \mathrm{mD}$ \\
$E_{3}$ & $2551 \mathrm{MPa}$ & $n_{0}^{\max }$ & $1.2 \mathrm{~mol} / \mathrm{L}$ & $\alpha_{h}$ & $0.135 \mathrm{MPa}^{-1}$ \\
$\nu$ & 0.198 & $P_{L}$ & $1.6 \mathrm{MPa}$ & $\alpha_{v}$ & $0.067 \mathrm{MPa}^{-1}$ \\
$\nu_{3}$ & 0.198 & $c$ & 9 & & \\
\hline
\end{tabular}

Details of the calibration work can be found in Espinoza et al. (2014), and the best fitting parameters are summarized in Table 1. Three points should be noted. First, the adsorptive properties given in Espinoza et al. (2014) were tested with $\mathrm{CO}_{2}$. For methane, we set $n_{0}^{\max }=1.2 \mathrm{~mol} / \mathrm{L}$ and $c=9$, which yields an adsorption stress for methane equal to about half of the adsorption stress for $\mathrm{CO}_{2}$ at the same pore pressure. Second, as discussed previously, the ratio $E / E_{3}$ can be greater or lower than 1 depending on the sample size. The tested coal cores in Espinoza et al. (2014) exhibited very little anisotropy. To assess the importance of the anisotropy of stiffness, we define a reference virtual fractured coal core that is more anisotropic. The vertical Young's modulus of the virtual material is identical to that of the tested coal cores $E_{3}=2551 \mathrm{MPa}$, but the horizontal Young's modulus is set such as $E / E_{3}=1.6$. Third, for the sake of simplification, the anisotropy of mechanical properties is assumed to totally come from the inequality between $E$ and $E_{3}$, so we set $\nu=\nu_{3}=0.198$.

\section{Analytical models for coal permeability changes}

\subsection{Analytical models for different geomechanical conditions}

For a representative volume element of fractured coal in oedometric condition i.e., $\Delta \varepsilon_{h}=0$ and $\Delta \sigma_{v}=0$, combing Equation (5) into the first three equations in Equation (1) yields:

$$
\begin{aligned}
\Delta \sigma_{h} & =\left[\frac{\nu_{3} E}{(1-\nu) E_{3}}+\frac{E}{3 K_{m}(1-\nu)}-1\right] \Delta P-\frac{E}{3 K_{m}(1-\nu)} \Delta S^{a} \\
\Delta \sigma_{v} & =0
\end{aligned}
$$

We recall that the stiffness coefficients as function of Young's modulus and of Poisson ratios for transverse isotropic case are: 


$$
\left\{\begin{aligned}
C_{11} & =\frac{E\left(\nu_{3}^{2} E-E_{3}\right)}{\left(\nu E_{3}-E_{3}+2 \nu_{3}^{2} E\right)(1+\nu)} \\
C_{12} & =\frac{-E\left(\nu_{3}^{2} E+\nu E_{3}\right)}{\left(\nu E_{3}-E_{3}+2 \nu_{3}^{2} E\right)(1+\nu)} \\
C_{13} & =\frac{-\nu_{3} E E_{3}}{\left(\nu E_{3}-E_{3}+2 \nu_{3}^{2} E\right)} \\
C_{33} & =\frac{(\nu-1) E_{3}^{2}}{\left(\nu E_{3}-E_{3}+2 \nu_{3}^{2} E\right)}
\end{aligned}\right.
$$

The variation of mean Terzaghi's effective stress is:

$$
\Delta\left(\frac{2 \sigma_{h}+\sigma_{v}+3 P}{3}\right)=\left[\frac{2 \nu_{3} E}{3(1-\nu) E_{3}}+\frac{2 E}{9 K_{m}(1-\nu)}+\frac{1}{3}\right] \Delta P-\frac{2 E}{9 K_{m}(1-\nu)} \Delta S^{a}
$$

Combing Equation (7) into Equation (6), we finally obtain the pressure-permeability relationship in oedometric condition:

$$
k=k_{0} \exp \left(\alpha_{h}\left(\left[\frac{2 \nu_{3} E}{(1-\nu) E_{3}}+\frac{2 E}{3 K_{m}(1-\nu)}+\frac{\alpha_{v}}{\alpha_{h}}\right] \Delta P-\frac{2 E}{3 K_{m}(1-\nu)} \Delta S^{a}\right)\right)
$$

In isochoric condition, $\Delta \varepsilon_{h}=\Delta \varepsilon_{v}=0$. Similar to the derivation in case of oedometric condition, the horizontal and vertical stresses in isochoric condition are:

$$
\begin{aligned}
& \Delta \sigma_{h}=-b_{1} \Delta P-\left(1-b_{1}\right) \Delta S^{a} \\
& \Delta \sigma_{v}=-b_{3} \Delta P-\left(1-b_{3}\right) \Delta S^{a}
\end{aligned}
$$

Then the pressure-dependent permeability in isochoric condition is:

$$
k=k_{0} \exp \left(\left[2 \alpha_{h}\left(1-b_{1}\right)+\alpha_{v}\left(1-b_{3}\right)\right]\left(\Delta P-\Delta S^{a}\right)\right)
$$

The equations of pressure-dependent permeability for different cases (Equations 10 and 12) comprise two terms: 1) variation of pressure, and 2) variation of adsorption stress. Actually, the two terms distinguish two mechanisms that govern changes in the cleat permeability. Consider reduction in pore pressure during depletion. The first term, called as a "poromechanical" term, leads to a cleat compression because the effective stress increases. The second term, called as an "adsorptive" term, causes a matrix shrinkage resulting from pressure decrease. The two terms have opposite effects: the "poromechanical" term results in a decrease in $k$, whereas the "adsorptive" term leads to an increase in $k$. The final $k-P$ curve is the combination of the two opposing effects (Figure 2). In oedometric condition, the curve exhibits a concave shape. For 

predominates so the cleat permeability ing predominates so the cleat permeability increases with decreasing pore pressure. However, for high pressure, $d S^{a} / d P$ reduces significantly (Figure 1b) so that the "poromechanical" term becomes predominant; the cleat permeability decreases with decreasing pressure. In isochoric condition, the cleat permeability vs. pressure is a monotonic function because the adsorption stress is always greater than the pore pressure in the studied range.

In oedometric condition, it exists a rebound pressure that marks the minimum value of the cleat permeability. The rebound pressure is obtained by solving for $\partial k / \partial P=0$, which yields:

$$
\frac{d S^{a}}{d P}=\left[\frac{2 \nu_{3} E}{(1-\nu) E_{3}}+\frac{2 E}{3 K_{m}(1-\nu)}+\frac{\alpha_{v}}{\alpha_{h}}\right] /\left(\frac{2 E}{3 K_{m}(1-\nu)}\right)
$$

For the reference material, Equation (13) yields $d S^{a} / d P=2.9$, and the rebound pressure is 7.2 MPa. From the equation above, one can deduce that the increase of $E / E_{3}$ leads to the increase of $d S^{a} / d P$, thus the rebound pressure decreases accordingly; while the decrease of $\alpha_{v} / \alpha_{h}$ results in an increase in the rebound pressure.

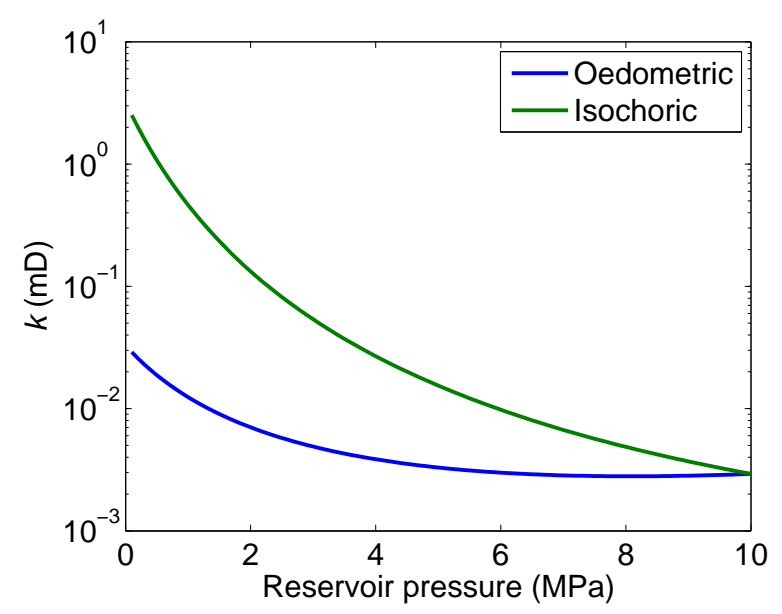

Figure 2: Evolution of permeability with reservoir pressure in oedometric and isochoric conditions at the REV scale.

\subsection{Model validation}

The oedometric condition is a common field condition assumed in the analytical models such as P-M model and S-D model. Broadly, our proposed model is a generalization of the relation between stresses and permeability in the S-D model: instead of only considering the dependence of permeability on the effective horizontal stress, as is the case in the S-D model, both the dependences on the horizontal and vertical 
stresses are considered in our model (see Equation 10). The fact that permeability depends also on vertical (i.e., axial) stresses and not only on horizontal (i.e., radial) ones has been experimentally shown by Espinoza et al. (2014). Moreover, the adsorption property of the coal matrix is described by a term of adsorption stress in our model instead of by a term of swelling strain in S-D model.

The proposed model is verified against both field and laboratory data. Shi and Durucan (2010) used their own model to fit the San Juan basin permeability data. The same data is also matched with our model (Equation 10). Most of the model parameters (as summarized in Table 1) are not available from the field data. However, as discussed previously, the main difference of our model in comparison with the S-D model lies in Equation (6), which also considers the effect of vertical stress on the permeability change. Hence, we focus here on this difference, and the anisotropy of the poromechanical properties are neglected. The Young modulus and Poisson ratios are assumed to be identical and chosen the same as those $\left(E=E_{3}=2900\right.$ $\left.\mathrm{MPa}, \nu=\nu_{3}=0.35\right)$ in Shi and Durucan (2010). The model parameters regarding the coal matrix $\left(K_{m}\right.$, $\left.n_{0}^{\max }, P_{L}, c\right)$ for the San Juan basin coal are assumed the same as those of the reference material (Table 1). Finally, only the two cleat compressibility constants are tuned to fit the field permeability data, such that, $\alpha_{h}=0.159 \mathrm{MPa}^{-1}, \alpha_{v}=0.001 \mathrm{MPa}^{-1}$. The model match is shown in Figure 3, as well as the match using the S-D model (Shi and Durucan, 2010). Broadly, the near-exponential increase growth of absolute permeability (up to nearly 10 fold) with reservoir drawdown from $5.5 \mathrm{MPa}$ is reproduced. The matched $\alpha_{h}$ value of $0.159 \mathrm{MPa}^{-1}$ is greater than that $\left(0.139 \mathrm{MPa}^{-1}\right)$ found with the S-D model (Shi and Durucan, 2010).

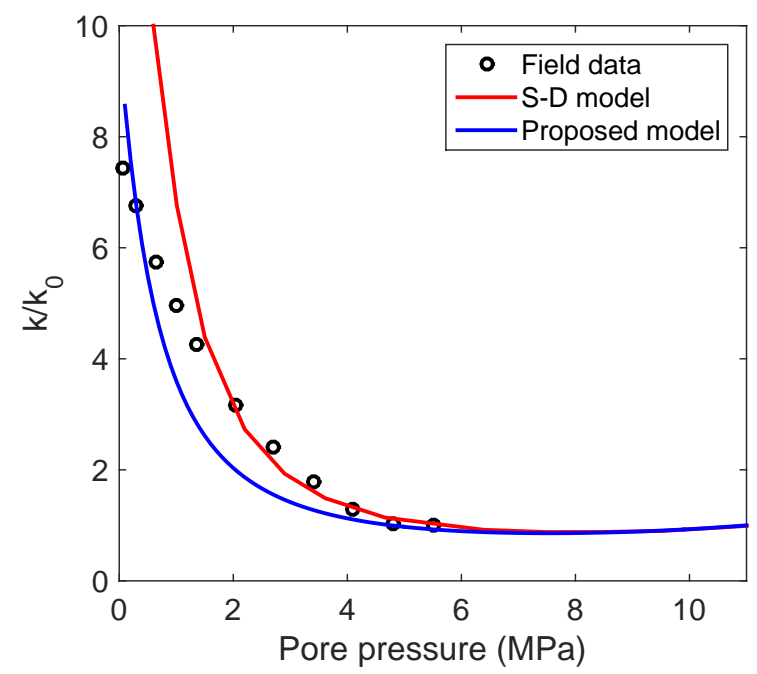

Figure 3: Model match of the San Juan basin permeability data (Shi and Durucan, 2010). The S-D model is also presented for comparison. 
The performance of the proposed model (Equations 7 and 10) is also tested using the laboratory data of Mitra et al., (2012), in which both the horizontal stress and the permeability are measured. As done in the model match for the field data, the parameters $K_{m}, n_{0}^{\max }, P_{L}$, and $c$ are assumed the same as those of the reference material (Table 1$)$. The Poisson ratios $\left(\nu, \nu_{3}\right)$ are assumed to be identical and equal to 0.3, i.e., the same as those in Shi and Durucan (2014), who also matched the laboratory data using their S-D model. Since the size of the samples in Mitra et al. (2012) is comparable to the size of the samples in Espinoza et al. (2014), we assume that $E=E_{3}$, as was approximately observed Espinoza et al. (2014) on their sample ${ }^{1}$. Finally, only three parameters are determined by fitting the experimental data, such that, $E=1450 \mathrm{MPa}$, $\alpha_{h}=0.344 \mathrm{MPa}^{-1}$, and $\alpha_{v}=0.037 \mathrm{MPa}^{-1}$. The determined values of $E$ and $\alpha_{h}$ are both within the range of available data (Liu et al., 2012). As shown in Figure 4, the proposed model can reproduce appropriately both the horizontal stress and the permeability. The deviation of the predicted horizontal stress for the lowest two pressure points (when $P$ is less than $1.4 \mathrm{MPa}$ ) can be explained by the reduction in Young's modulus at low pore pressure (Shi et al., 2014; Shi and Durucan, 2018).

The same experimental data has also been matched by Shi et al. (2014) using their own model. Applying a constant $\alpha_{h}$ value of $0.194 \mathrm{MPa}^{-1}$, the S-D model well reproduced the laboratory results when pore pressure varied from 6.2 MPa to 1.4 MPa but failed to match the data when the pore pressure reduced below 1.4 MPa (Figure 4). In contrast, the proposed model succeeds in fitting the whole experimental data using a constant $\alpha_{h}$ value.

\section{The role of anisotropy}

\subsection{Analytical modeling at the scale of representative volume element}

\subsubsection{The role of the anisotropy of stiffness}

In the following, the role of different anisotropy terms on the $k$ - $P$ relationship will be explored. To do so, three types of equivalent materials are established: the first neglecting the anisotropy of stiffness, the second neglecting the anisotropy of permeability dependence on stresses, and the third neglecting both anisotropies. The coefficients of these equivalent materials are calibrated on the permeability variations predicted with the anisotropic model (Figure 2). Comparisons are conducted to assess how well the equivalent isotropic models can reproduce the permeability variations predicted with the anisotropic model, and thus the role of the different types of anisotropy can be discussed.

\footnotetext{
${ }^{1}$ the anisotropy of the elastic properties is indeed expected to be somehow related to the size of the sample, since, in particular because of the presence of cleats, elastic properties are known to depend on the size of the sample
} 


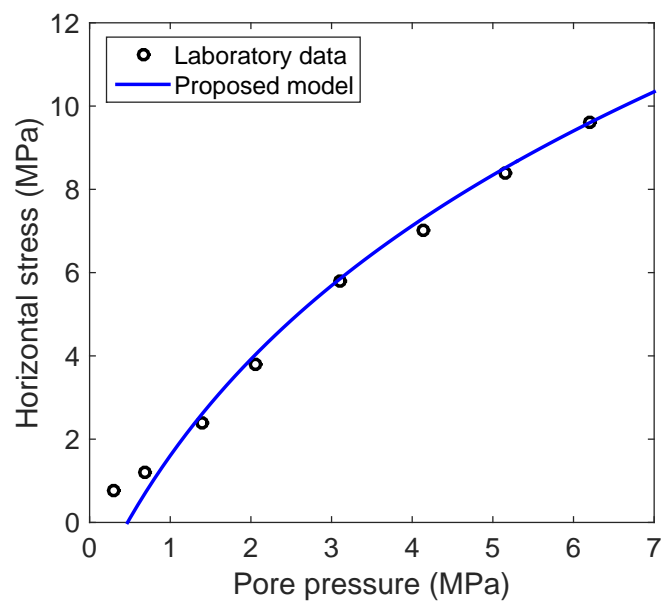

(a)

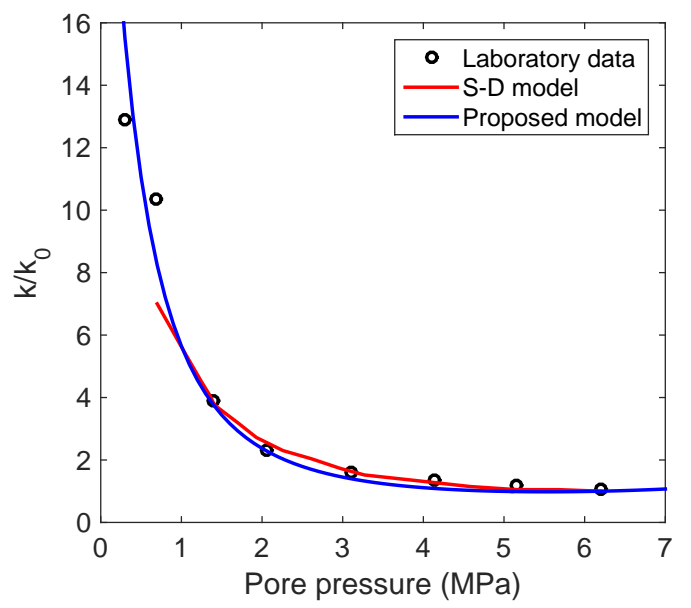

(b)

Figure 4: Validation of the proposed model using laboratory data of (Mitra et al., 2012): a) horizontal stress, b) permeability. The S-D model (Shi et al., 2014) is also presented for comparison.

As mentioned previously, the anisotropy of stiffness in the present work specifically refers to the difference between $E$ and $E_{3}$. To evaluate its importance for the $k$ - $P$ relationship, we consider an equivalent isotropic material. The two elastic constants are assessed in two ways. In the first way (called free $K$ method), the Young's modulus and Poisson ratio of the equivalent isotropic material are calculated such that its volumetric Terzaghi's effective stress in oedometric condition (refer to Equation 9) is identical to that of anisotropic material:

$$
\begin{aligned}
\frac{\nu^{i a}}{1-\nu^{i a}} & =\frac{\nu_{3} E}{(1-\nu) E_{3}} \\
\frac{E^{i a}}{\left(1-\nu^{i a}\right)} & =\frac{E}{(1-\nu)}
\end{aligned}
$$

We obtain $E^{i a}=3648 \mathrm{MPa}$ and $\nu^{i a}=0.283$. The superscript $i a$ stands for the equivalent material with isotropic stiffness and anisotropic permeability dependence on stresses. For this isotropic material, still employing the same anisotropic permeability dependence on stresses as for the reference anisotropic material, we predict exactly the same $k-P$ curve with respect to the reference anisotropic material in oedometric condition, shown in Figure 5a. Actually, in oedometric condition, the equivalence of the volumetric Terzaghi's effective stress (i.e., $2 \sigma_{h} / 3+P$ ) implies the equivalence of the horizontal stress. Hence, the anisotropy of stiffness can be neglected without introducing any error. However, using the free $K$ method, the bulk modulus of the isotropic material $\left(1 / K^{i a}=3(1-\nu) / E-3 \nu_{3} / E_{3}\right)$ is skewed (the isotropic bulk modulus of the anisotropic materials is $\left.1 / K=2(1-\nu) / E+\left(1-4 \nu_{3}\right) / E_{3}\right)$, shown in Figure 6 . To avoid the mismatch 
in bulk modulus, we propose another method (called constrained $K$ method): the bulk modulus $K$ is constrained, and the second elastic constant $\left(\nu^{i a}\right)$ is obtained by fitting the pressure-permeability curve in oedometric condition, i.e., minimizing $\left(\log k_{\mathrm{iso}}^{s}-\log k\right)^{2}$. The fitting yields $\nu^{i a}=0.219$. This constrained $K$ method can result in an almost perfect reproduction of the $P-k$ curve with respect to the reference anisotropic material (see Figure 5a).

To quantify the error induced by disregarding different terms of anisotropy, we define an error norm i.e., $e$ $=\operatorname{mean}\left(\left|\log \left(k_{\text {iso }}^{i} / k\right)\right|\right)$, where $i$ might refer to $s$ (in case of disregarding the anisotropy of stiffness), $p$ (in case of disregarding the anisotropy of permeability dependence on stresses) or $s p$ (in case of disregarding both anisotropies of stiffness and of permeability dependence on stresses). We carry out a parametric analysis varying $\alpha_{h}$ and $E$ with constrained $\alpha_{v}$ and $E_{3}$, and the results in case of disregarding the anisotropy of stiffness are shown in Figure 7, for both oedometric and isochoric conditions. In general, both methods reproduce well the pressure-permeability curve in oedometric condition, for which the averaged $k_{i s o}^{s} / k$ is less than $10^{0.05}=1.1$. Using the constrained $K$ method, the error increases with the anisotropy degree of stiffness and of permeability dependence on stresses.

The elastic constants of equivalent isotropic material calibrated in oedometric condition are applied for the prediction of the $P-k$ curve in isochoric condition, shown in Figure 5b. In general, the free $K$ method overestimates the cleat permeability, whereas the constrained $K$ method underestimates the cleat permeability. This trend can be explained by Equation 12, considering $b^{i a}$ equal to 0.44 and 0.58 for the free and constrained $K$ methods, respectively (compared to $b_{1}=0.52$ and $b_{3}=0.64$ ). Regarding the absolute value of the errors, the constrained $K$ method provides better prediction in isochoric condition: the average of $k_{i s o}^{s} / k$ is $10^{0.25}=1.8$ using the constrained $K$ method; whereas, it can reach $10^{0.60}=4.0$ using the free $K$ method (Figure $7 \mathrm{c}$ and $7 \mathrm{~d}$ ).

\subsubsection{The role of the anisotropy of permeability dependence on stresses}

We now evaluate the importance of the anisotropy of permeability dependence on stresses. To do so, while keeping the anisotropic elastic properties unchanged, we consider a permeability that only depends on the volumetric stress so that the two coefficients $\alpha_{h}$ and $\alpha_{v}$ are replaced by a single coefficient $\alpha^{a i}$. This coefficient is determined by fitting the $P$ - $k$ curve using the same principle as for the identification of the second elastic constant of the equivalent isotropic material using constrained $K$ method: it yields $\alpha^{a i}$ $=0.156 \mathrm{MPa}^{-1}$. For this virtual material that obeys isotropic permeability dependence on stresses, the pressure-permeability curve is below that for the reference material in oedometric condition (Figure 5c); whereas, the permeability is overestimated in isochoric condition (Figure $5 \mathrm{~d}$ ). The fitted value $\alpha^{a i}$ depends 
on $\alpha_{h} / \alpha_{v}$ as well as $E / E_{3}$ : it may be beyond the $\left[\alpha_{v}, \alpha_{h}\right]$ interval; it can be even negative in some cases (Figure 8).

The error $e$ on permeability induced when disregarding the anisotropy of permeability dependence on stresses is shown in Figure 9. It is normal to observe that the error increases with $\alpha_{h} / \alpha_{v}$. The dependence of the error with $E / E_{3}$ is more complex. In oedometric condition, the error increases with $E / E_{3}$ up to a maximum and then reduces slightly (Figure $9 \mathrm{~b}$ ). In isochoric condition, two troughs exist in the $e-E / E_{3}$ curve: the first is at lowest $E / E_{3}$ value, while the second is close to 1.4 with $e=0$ regardless of the $\alpha_{h} / \alpha_{v}$ value (Figure 9d).

Compared to neglecting the anisotropy of stiffness, disregarding the anisotropy of permeability leads to larger deviations from the $k-P$ curves in the anisotropic case: in the tested $\alpha_{h} / \alpha_{v}$ and $E / E_{3}$ intervals, the averaged $k_{\mathrm{iso}}^{p} / k$ in oedometric and isochoric conditions can reach nearly $10^{0.3}=2.0$ and $10^{1.2}=15.8$, respectively, in comparison to $10^{0.05}=1.1$ and $10^{0.25}=1.8$ when neglecting the anisotropy of stiffness.

\subsubsection{Disregarding both anisotropies of stiffness and of permeability dependence on stresses}

We now consider the case where both anisotropies of stiffness and of permeability dependence on stresses are neglected. In this totally isotropic case, the material properties reduce to three: one for the permeability law $\left(\alpha^{i i}\right)$ and two for the stiffness $\left(E^{i i}\right.$ and $\left.\nu^{i i}\right)$. To assess the three parameters, a method comparable to that used for investigating the role of the anisotropy of stiffness is applied again: the two coefficients before the pore pressure and adsorption stress terms are identical in anisotropic and totally isotropic cases for oedometric condition (Equation 10). Since the oedometric condition only leads to two equations for three unknowns, the system is underdetermined. The third equation can be introduced by equating the $P-k$ curves in isochoric condition (Equation 12). Thus,

$$
\begin{aligned}
\frac{2 \nu_{3} E \alpha_{r}}{(1-\nu) E_{3}}+\frac{2 E \alpha_{r}}{3 K_{m}(1-\nu)}+\alpha_{a} & =\frac{2 \nu^{i i} \alpha^{i i}}{1-\nu^{i i}}+\frac{2 E^{i i} \alpha^{i i}}{3 K_{m}\left(1-\nu^{i i}\right)}+\alpha^{i i} \\
\frac{2 E \alpha_{r}}{3 K_{m}(1-\nu)} & =\frac{2 E^{i i} \alpha^{i i}}{3 K_{m}\left(1-\nu^{i i}\right)} \\
2 \alpha_{r}\left(1-b_{1}\right)+\alpha_{a}\left(1-b_{3}\right) & =3 \alpha^{i i}\left(1-b^{i i}\right)
\end{aligned}
$$

For the totally isotropic material, Equation (15) yields $E^{i i}=4342 \mathrm{MPa}, \nu^{i i}=0.099$, and $\alpha_{i i}=0.142$ $\mathrm{MPa}^{-1}$. This leads to an exact reproduction of the $k-P$ curves in both oedometric and isochoric conditions (Figures 5e and 5f). However, as for the case of disregarding the anisotropy of stiffness, the bulk modulus of the totally isotropic material is skewed respect to the bulk modulus of the reference anisotropic material (Figure 10). Moreover, this equivalent totally isotropic material cannot reproduce the same $P$ - $k$ curves in 
other geomechanical conditions, and the error might be more significant than for the case when only one term of anisotropy is neglected. We provide here two examples: free swelling condition (i.e., $\Delta \sigma_{r}=\Delta \sigma_{a}=$ $0)$ and inverse oedometric condition (i.e., $\Delta \sigma_{r}=0, \Delta \varepsilon_{a}=0$ ), shown in Figure 11.

\subsection{Numerical simulation at the scale of reservoir}

In the section above, the role of anisotropy in permeability changes was investigated at the scale of representative element volume, and some geomechanical assumptions were employed in the derivation of the analytical equations, e.g., the assumption of uniaxial strain and constant vertical stress (i.e. of oedometric condition). These assumptions do not exactly match the coalbed conditions for real methane recovery at the scale of reservoir, and so the accuracy of the analytical modeling should be investigated. For instance, oedometric condition is the most common condition used for matching CBM production (Palmer and Mansoori, 1998; Shi and Durucan, 2004). During the production, subsidence and pressure gradients lead to creation of shear stress as well as changes in vertical stress (Settari, 2002). Moreover, there is field evidence that the uniaxial strain assumption may not be accurate during reservoir subsidence (Connell, 2009).

This section is devoted to assessing how formation permeability changes with declining reservoir pressure. Once the pressure-permeability relationship is established independently, the analytical models and subsequent analysis obtained in the previous section can be tested in how well they match the response at the reservoir scale. To do so, reservoir simulations are performed considering the same four materials as in the previous section. 1) A totally anisotropic reference material with material properties given in Table 1. 2) An equivalent isotropic material, but the permeability of which still depends in different manners on horizontal and vertical effective stresses. The isotropic mechanical constants are $E^{i a}=3648 \mathrm{MPa}$ and $\nu^{i a}=$ 0.283. 3) An anisotropic material, but with the permeability that only depends on the volumetric stress: its cleat compressibility is $\alpha_{a i}=0.156 \mathrm{MPa}^{-1}$. 4) A fully isotropic material: its elastic properties are isotropic $\left(E^{i i}=4342 \mathrm{MPa}\right.$ and $\left.\nu^{i i}=0.099\right)$, and its permeability only depends on the volumetric stress $\left(\alpha^{i i}=0.142\right.$ $\left.\mathrm{MPa}^{-1}\right)$.

We model the depletion of a coal seam containing pure methane at $312.15 \mathrm{~K}$. Vertical and horizontal insitu total stresses are $23 \mathrm{MPa}$ and $14 \mathrm{MPa}$, respectively; initial pressure of methane is $10 \mathrm{MPa}$. Simulation is axisymmetrical. The radius of the borehole is $0.1 \mathrm{~m}$. The radius of the coal seam is $100 \mathrm{~m}$, its thickness is 5 m. 30-m-thick impervious isotropic cap rock and bed rock are considered in the simulation, their mechanical properties are: $E_{c}=10 \mathrm{GPa}$ and $\nu_{c}=0.26$. The simulation is conducted through the in-house software "Bil" based on the finite element method. The mesh (with a total element number of 21571) used in the simulations is displayed in Figure 12, together with the mechanical boundary conditions. With regards to 
the hydraulic boundary conditions in the coal seam, flow is null at outer boundary. Two types of boundary conditions are considered at the borehole: constant production rate $\left(0.001 \mathrm{~mol} /\left(\mathrm{s} . \mathrm{m}^{2}\right)\right)$ and constant pressure (1 MPa). In case of constant production rate, the small rate is employed so that methane can be recovered more fully and the reservoir pressure can fall to a low value (down to $5 \mathrm{MPa}$ in our simulation) to maintain this production rate. This enables obtaining the permeability variation curve in a wide pressure range. We consider that the kinetics of methane flowing from the coal matrix to the cleats is very fast: at any time, at any location in the coal seam, the pressure of the fluid in the cleats and the thermodynamic pressure of the fluid in the coal matrix are considered to be equal.

\subsubsection{Estimation of reservoir permeability}

The reservoir simulation gives rise to production data including gas rate history, evolution of pressure profile in the reservoir. These data are then used to calculate the formation permeability $k_{g}(\mathrm{in} \mathrm{mD})$ using the production data analysis method (Lee et al., 1984; Clarkson, 2007):

$$
k_{g}=\frac{q_{g} T\left[\ln \left(r_{e} / r_{w}\right)-0.5+s+D q_{g}\right]}{7.03 \times 10^{-4} h\left[m\left(P_{r}\right)-m\left(P_{w f}\right)\right]}
$$

where $r_{e}$ and $r_{w}$ are drainage radius and wellbore radius, $q_{g}$ is gas surface flow rate, $m$ is pseudo-pressure (in $\mathrm{psi}^{2} / \mathrm{cp}$ ), $P_{r}$ and $P_{w f}$ stand for reservoir pressure and bottomhole pressure, $s$ is skin factor (assumed equal to 0 here), and $D$ is inertial or turbulent flow factor (in D/Mscf, assumed equal to 0 here). In the following, the reservoir pressure $P_{r}$ is equal to the pressure at the outer boundary $P_{e}$. The derivation of Equation (16) is based on the pseudo-steady-state analytical solution of a radial flow from a finite cylindrical reservoir at a constant production rate, and the derivation details are provided in Appendix A. It should be noted that our equation slightly differs from the equation in the literature (Lee et al., 1984; Clarkson, 2007): the constant 0.75 is modified into 0.5 .

To validate Equation (16), we model recovery of methane from a coal seam with invariant permeability (i.e., $\alpha_{h}=\alpha_{v}=0$ ). The other mechanical and permeability properties are the same as the reference material. Equation (16) is valid for the pseudo-steady-state, i.e., $t_{D}>0.3 r_{e D}^{2}$ (refer to Appendix A). For the considered system, the time for reaching the pseudo-steady-state is 4.5 years. After this time, the estimated reservoir permeability agrees well with the coal permeability, which validates Equation (16).

\subsubsection{Reservoir simulation results}

The profiles of pore pressure at different times in two cases (i.e., constant flow and constant pressure) are shown in Figure 13; the evolution of borehole pressure and pressure at outer boundary is displayed in 
Figure 14. Given the range of applicability of Equation (16), the pressure-permeability curve is estimated only after 4.5 years or more have elapsed since methane production started. The $k-P$ curve determined from the reservoir simulation is shown in Figure 15. The analytical model curves for oedometric and isochoric conditions also are given for comparison. One observes that oedometric condition gives a good representation of the reservoir. Compared to the constant-pressure condition, the constant-flow condition gives rise to a $k-P$ curve more consistent with that for the analytical oedometric condition. This is mainly because Equation (16) is derived from the analytical solution of the flow equation in case of constant production rate.

When the anisotropic material is simplified by an equivalent material with isotropic stiffness using Equation (14), we reproduce the same $k_{g}$-P curve as in the reference anisotropic case. This is also the case when both anisotropies of stiffness and of permeability dependence on stresses are disregarded. When neglecting the anisotropy of permeability dependence on stresses, the pressure-dependent permeability curve deviates from the curve for the anisotropic material: for the reference material considered, it underestimates the reservoir permeability. These observations at the scale of reservoir are comparable with those obtained from analytical modeling at the scale of representative element volume.

\section{Conclusion}

The effect of anisotropy on the horizontal permeability changes in coal seams was investigated. The analysis was based on a fully anisotropic model consisting of both the anisotropies of stiffness and of how horizontal permeability depends on stress. The main findings include:

- The analytical expression of the horizontal permeability changes in oedometric condition is derived. Different from the existing models that merely considered the dependence of the horizontal permeability on the horizontal stress or on the mean stress, the proposed model separately incorporates the effects of the horizontal effective stress and of the vertical effective stress. Moreover, the mechanical anisotropy is also taken into account. The proposed model is validated against both available laboratory and field data.

- On the basis of the analytical expression, we demonstrate that the mechanically anisotropic materials can be simplified by an isotropic material without introducing any error in permeability changes (called free $K$ method). However, this free $K$ method leads to a fitted bulk modulus that differs from the actual bulk modulus of the material, and results in significant errors on the permeability variations estimated in isochoric condition. Hence, an alternative method with constrained $K$ (i.e., with a bulk 


\section{Appendix A. Estimation of permeability-pressure relationship from production data}

Consider a radial flow from a finite cylindrical reservoir (with sealed upper and lower surfaces) at a constant production rate. The flow at the outer boundary is zero at all times. The flow equation for a cylindrical flow described in terms of dimensionless variables is (Board, 1979):

$$
\frac{1}{r_{D}} \frac{\partial}{\partial r_{D}}\left[r_{D} \frac{\partial}{\partial r_{D}}\left(\Delta P_{D}\right)\right]=\frac{\partial}{\partial t_{D}}\left(\Delta P_{D}\right)
$$




$$
\left.r_{D} \frac{\partial}{\partial r_{D}}\left(\Delta P_{D}\right)\right|_{r_{D}=1}=-1
$$

b) The flow at the outer boundary is zero,

$$
\left.\frac{\partial}{\partial r_{D}}\left(\Delta P_{D}\right)\right|_{r_{e D}}=0
$$

c) The initial reservoir pressure is uniform,

$$
\Delta P_{D}=0
$$

The dimensionless variables are defined as:

$$
\begin{gathered}
r_{D}=\frac{r}{r_{w}} \\
t_{D}=\frac{\lambda k t}{\phi \mu_{i} c_{i}^{f} r_{w}^{2}} \\
\Delta P_{D}=\frac{m_{i}-m}{m_{i} q_{D}} \\
q_{D}=\frac{\gamma T q_{s t}}{k h m_{i}}
\end{gathered}
$$

where $k$ is permeability, $\phi$ is porosity, $\mu$ is viscosity of fluid, $c^{f}$ is compressibility of fluid, $h$ is the formation thickness. The subscript $i$ stands for the initial condition. The two coefficient $\lambda$ and $\gamma$ include coefficient like 2 and $\pi$, units conversion factors, and the numerical values of $P_{s t}$ and $T_{s t}$ that may be inherent in the definitions of the dimensionless terms. The pseudo-pressure $m$ is defined as:

$$
m=2 \int_{P_{0}}^{P} \frac{P}{\mu Z} d P
$$

where $P_{0}$ is a specified reference pressure. The compressibility factor, $Z$, is a correlation factor which defines the deviation of a real gas from ideal gas behaviour.

If the well radius can be assumed to be vanishingly small (i.e., $r_{e} \gg r_{w}$ ), the general solution of the problem is: 
where $\alpha_{n}$ are the roots of

$$
J_{1}\left(\alpha_{n} r_{e D}\right) Y_{1}\left(\alpha_{n}\right)-J_{1}\left(\alpha_{n}\right) Y_{1}\left(\alpha_{n} r_{e D}\right)=0
$$

$J_{1}$ and $Y_{1}$ are Besel functions of the first and second kind, respectively and both of order one.

When $t_{D}$ is sufficient large $\left(t_{D}>0.3 r_{e D}^{2}\right)$, the summation term in Equation A.10) becomes negligible, and the pressure at the well and at the outer boundary are simply:

$$
\begin{gathered}
\left.\Delta P_{D}\right|_{r_{D}=1}=\frac{2 t_{D}}{r_{e D}^{2}}-0.75+\ln r_{e D} \\
\left.\Delta P_{D}\right|_{r_{e D}}=\frac{2 t_{D}}{r_{e D}^{2}}-0.25
\end{gathered}
$$

Combining the two above equations and using the definition of the dimensionless variables, we obtain,

$$
m\left(P_{r}\right)-m\left(P_{w f}\right)=\frac{\gamma T q_{s t}}{k h}\left(\ln r_{e D}-0.5\right)
$$

In the derivation above, it was assumed that the medium is homogeneous and isotropic and that flow is single-phase and obeys Darcy's law. In real case, deviations from these idealizations are frequent and can not be ignored. Two terms commonly are considered: 1) the skin effect to account for the altered permeability at the vicinity of well because of well drilling, fracturing or acidizing on completion; 2) the inertial / turbulent flow effect that are not taken into account by Darcy's law. Taking the two effects into account, we finally obtain the equation for estimating the formation permeability by production data.

\section{References}

Board, E.R.C., 1979. Gas Well Testing: Theory and Practice. Energy Resources Conservation Board.

Brochard, L., Vandamme, M., Pellenq, R.J.M., 2012. Poromechanics of microporous media. Journal of Mechanics and Physics of Solids 60, 606-622.

Cheng, A.D., 1997. Material coefficients of anisotropic poroelasticity. International Journal of Rock Mechanics and Mining Science 34, 199-205.

Clarkson, C.R., Jordan, C.L., Gierhart, R.R., Seidle, J.P., 2007. Production data analysis of CBM wells. SPE107705. Coussy, O., 2010. Mechanics and Physics of Porous Solids. Wiley. 

from deep coal seams. American Association of Petroleum Geologists Bulletin 89 (9), 1181-1202.

Cui, X., Bustin, R.M., Chikatamarla, L., 2007. Adsorption-induced coal swelling and stress: implications for methane production and acid gas sequestration into coal seams. Journal of Geophysical Research 112, B10202.

Connell, L.D., 2009. Coupled flow and geomechanical processes during gas production from coal seams. International Journal of Coal Geology 79, 18-28.

Day, S., Fry, R., Sakurovs, R., 2008. Swelling of Australian coals in supercritical $\mathrm{CO}_{2}$. International Journal of Coal Geology $74,41-52$.

Espinoza, D.N., Vandamme, M., Dangla, P., Pereira, J.M., Vidal-Gilbert, S., 2013. A transverse isotropic model for microporous solids: Application to coal matrix adsorption and swelling. Journal of Geophysical Research: Solid Earth 118(12), 6113-6123.

Espinoza, D.N., Vandamme, M., Pereira, J.M., Dangla, P., Vidal-Gilbert, S., 2014. Measurement and modeling of adsorptiveporomechanical properties of bituminous coal cores exposed to $\mathrm{CO}_{2}$ : adsorption, swelling strains, swelling stresses and impact on fracture permeability. International Journal of Coal Geology 134-135, 80-95.

Hol, S., Spiers, C.J., 2012. Competition between adsorption-induced swelling and elastic compression of coal at CO 2 pressures up to100MPa. Journal of the Mechanics and Physics of Solids 60, 1862-1882.

Laubach, S.E., Marrett, R.A., Olson, J.E., Scott, A.R., 1998. Characteristics and origins of coal cleat: a review. International Journal of Coal Geology 35, 175-207.

Lee, W.J., Kuo, T.B., Holditch, S.A., McVay, D.A., 1984. Estimating formation permeability from single-point flow data, SPE/DOE/GRI12847.

Liu, S., Harpalani, S., Mitra, A., 2012. Laboratory measurement and modelling of coal permeability with continued methane production: part 2 - Modelling results. Fuel 94, 117-124.

Mitra, A., Harpalani, S., Liu, S.M., 2012. Laboratory measurement and modeling of coal permeability with continued methane production: Part 1 - Laboratory results. Fuel 94, 110-116.

Morcote, A., Mavko, G., Prasad, M., 2010. Dynamic elastic properties of coal. Geophycis 75, E227-E234.

Nikoosokhan, S., Vandamme, M., Dangla, P., 2014. A poromechanical model for coal seams saturated with binary mixtures of $\mathrm{CH}_{4}$ and $\mathrm{CO}_{2}$. Journal of the Mechanics and Physics of Solids 71, 97-111.

Palmer, I., 2009. Permeability changes in coal: analytical modeling. International Journal of Coal Geology 77, $119-126$.

Palmer, I., Mansoori, J., 1998. How permeability depends on stressand pore pressure in coalbeds: a new model. SPE Reservoir Evaluation \& Engineering, 539-544.

Palmer, I.D., Mavor, M., Gunter, B., 2007. Permeability changes in coal seams during production and injection. International Coalbed Methane Symposium. University of Alabama, Tuscaloosa, Alabama. Paper 0713.

Pan, Z.J., Connell, L.D., 2007. A theoretical model for gas adsorption-induced coal swelling. International Journal of Coal Geology 69, 243-252.

Pan, Z.J., Connell, L.D., 2011. Modelling of anisotropic coal swelling and its impact on permeability behaviour for primary and enhanced coalbed methane recovery. International Journal of Coal Geology 85, 257-267.

Peng, Y., Liu, J., Wei, M., Pan, Z., Connell, L.D., 2014. Why coal permeability changes under free swellings: New insights. International Journal of Coal Geology, 133, 35-46.

Pone, J.D.N., Halleck, P.M., Mathews, J.P., 2010. 3D characterization of coal strains induced by compression, carbon dioxide sorption, and desorption at in-situ stress conditions. International Journal of Coal Geology 82, 262-268. 
Seidle, J.P., Jeansonne, M.W., Erickson, D.J., 1992. Application of matchstick geometry to stress dependent permeability in coals. Rocky Mountain Regional Meeting of the Society of Petroleum Engineers. Casper, Wyoming. SPE 24361.

Settari, A., 2002. Reservoir compaction. Journal of Petroleum Technology 54(8), 62-69.

Shi, J.Q., Durucan, S., 2004. Drawdown induced changes in permeability of coalbeds: a new interpretation of the reservoir response to primary recovery. Transport in Porous Media 56(1), 1-16.

Shi, J.Q., Durucan, S., 2010. Exponential growth in San Juan basin Fruitland coalbed permeability with reservoir drawdown: model match and new insights. SPE Reservoir Evaluation \& Engineering 13(6), 914-925.

Shi, J.Q., Durucan, S., 2014. Modelling laboratory horizontal stress and coal permeability data using S\&D permeability model. International Journal of Coal Geology 131, 172-176.

Shi, J.Q., Pan, Z., Durucan, S., 2014. Analytical models for coal permeability changes during coalbed methane recovery: Model comparison and performance evaluation, International Journal of Coal Geology 136, 17-24.

Shi, J.Q., Durucan, S., 2018. Variation in horizontal stress with pore pressure depletion in coal under uniaxial strain conditions: An update on the modelling of laboratory data, International Journal of Coal Geology 187, 94-97.

Somerton, W.H., Soylemezolu, I.M., Dudley, R.C., 1975. Effect of stress on permeability of coal. Journal of Rock Mechanics and Mining Science \& Geomechanics Abstracts 12, 129-145.

Vandamme, M., Brochard, L., Lecampion, B., Coussy, O., 2010. Adsorption and strain: the $\mathrm{CO}_{2}$-induced swelling of coal. Journal of Mechanics and Physics of Solids 58, 1489-1505.

Wang, G.X., Massarotto, P., Rudolph, V., 2009. An improved permeability model of coal for coalbed methane recovery and $\mathrm{CO}_{2}$ geosequestration. International Journal of Coal Geology 77, 127-136.

Wang, J.G., Liu, J.S., Kabirc, A., 2013. Combined effects of directional compaction, non-Darcy flow and anisotropic swelling on coal seam gas extraction. International Journal of Coal Geology 109-111, 1-14.

Wang, K., Zang, J., Wang, G.D., Zhou, A.T., 2014. Anisotropic permeability evolution of coal with effective stress variation and gas sorption: Model development and analysis. International Journal of Coal Geology 130, 53-65. 
Glossary

$b_{1}, b_{3}$ : Horizontal and vertical Biot coefficient [-]

$c$ : Adsorption-strain coupling coefficient [-]

$C_{i j}$ : Stiffness tensor coefficient defined at the coal seam scale $[\mathrm{Pa}]$

$D$ : Inertial or turbulent flow factor [D/Mscf]

$E, E_{3}$ : Horizontal and vertical Young's modulus $[\mathrm{Pa}]$

$h$ : Formation thickness [ft]

$k$ : Coal horizontal permeability $[\mathrm{mD}]$

$k_{0}$ : Reference coal horizontal permeability (at $10 \mathrm{MPa}$ ) $[\mathrm{mD}]$

$k_{g}$ : Reservoir horizontal permeability $[\mathrm{mD}]$

$K$ : Coal seam bulk modulus $[\mathrm{Pa}]$

$K_{m}$ : Coal matrix bulk modulus $[\mathrm{Pa}]$

$m(P):$ Pseudopressure $\left[\mathrm{psi}^{2} / \mathrm{cp}\right]$

$n_{m}$ : Fluid amount in the coal matrix per unit volume of coal matrix $[\mathrm{mol} / \mathrm{L}]$

$n_{0}^{\max }:$ Asymptotic parameter of constant matrix volume Langmuir isotherm $[\mathrm{mol} / \mathrm{L}]$

$n_{T}$ : Total fluid amount per unit volume of coal seam REV [mol/L]

$N$ : Coal seam Biot modulus [Pa]

$P$ : Pore pressure $\left(P=P_{c}=P_{m}\right)[\mathrm{Pa}]$

$P_{c}$ : Pressure of fluid in the cleat $[\mathrm{Pa}]$

$P_{e}$ : Pressure at the outer boundary in the simulation $[\mathrm{Pa}]$

$P_{L}$ : Langmuir pressure of constant matrix volume Langmuir isotherm $[\mathrm{Pa}]$

$P_{m}$ : Thermodynamical pressure of fluid in the coal matrix $[\mathrm{Pa}]$

$P_{r}:$ Reservoir pressure [psia]

$P_{w f}:$ Flowing bottomhole pressure [pisa]

$q_{g}$ : Gas surface flow rate $[\mathrm{Mscf} / \mathrm{D}]$

$r_{e}$ : Drainage radius [ft]

$r_{w}$ : Wellbore radius [ft]

$s$ : Skin factor [-]

$S^{a}$ : Coal matrix adsorption stress $[\mathrm{Pa}]$

$T$ : Temperature $\left[{ }^{\circ} \mathrm{R}\right]$

$U$ : Chemical potential $[\mathrm{J} / \mathrm{mol}]$ 
$V_{b}$ : Molar volume of the fluid in bulk conditions $[\mathrm{L} / \mathrm{mol}]$

Subscript

1,2: Horizontal

3: Vertical

$D$ : Dimensionless

$h$ : Horizontal direction

$v$ : Vertical direction

Superscript

$i a$ : In case of isotropic stiffness and anisotropic permeability dependence on stresses $a i$ : In case of anisotropic stiffness and isotropic permeability dependence on stresses $i i$ : In case of isotropic stiffness and isotropic permeability dependence on stresses Greek symbols

$\alpha$ : Cleat compressibility coefficient for permeability law $\left[\mathrm{MPa}^{-1}\right]$

$\varepsilon_{m}$ : Volumetric strain of the coal matrix [-]

$\varepsilon_{i j}$ : Strain tensor defined at the coal seam scale [-]

$\phi_{c}$ : Cleat porosity or macroporosity [-]

$\mu$ : Viscosity of fluid [cp]

$\nu, \nu_{3}$ : Horizontal and vertical Poisson ratios [-]

$\sigma_{i j}$ : Total stress tensor defined at the coal seam scale [-]

$\rho_{b}$ : Bulk molar density of the fluid $[\mathrm{mol} / \mathrm{L}]$

Units conversion

$1 \mathrm{ft}=0.3048 \mathrm{~m}$

$1 \mathrm{psi}=6895 \mathrm{~Pa}$

$1 \mathrm{cp}=0.001$ Pa.s 


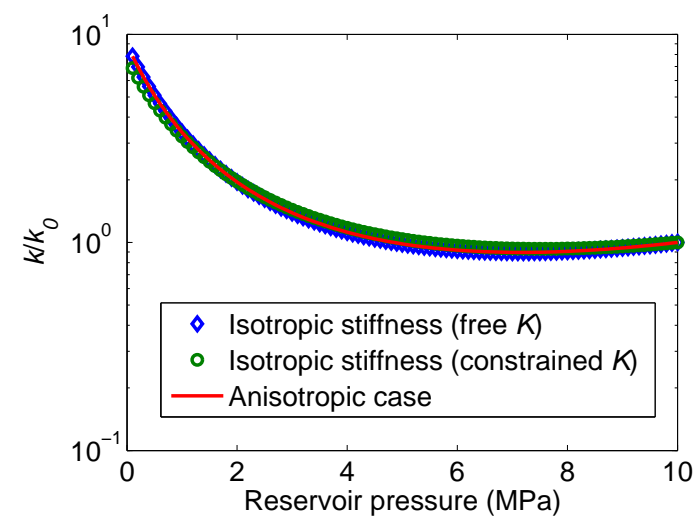

(a)

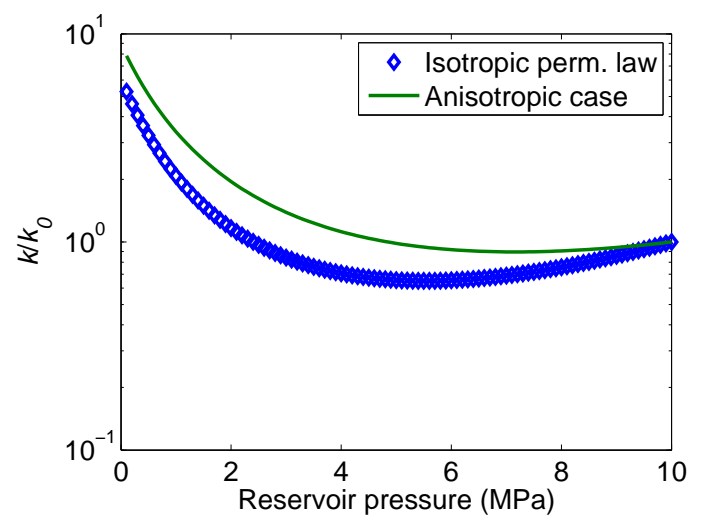

(c)

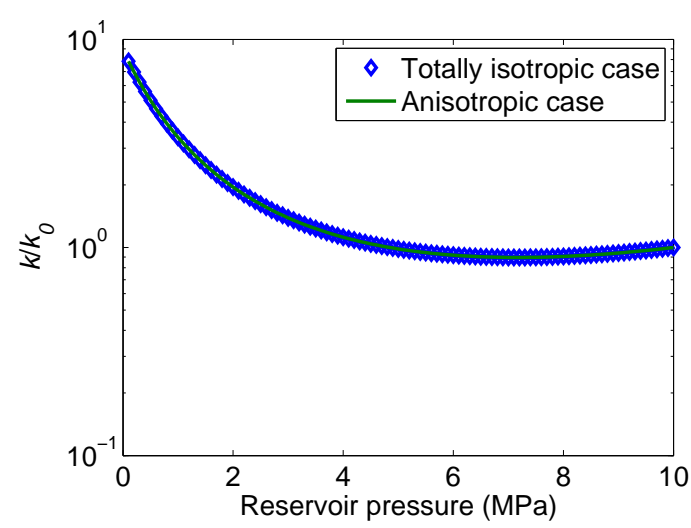

(e)

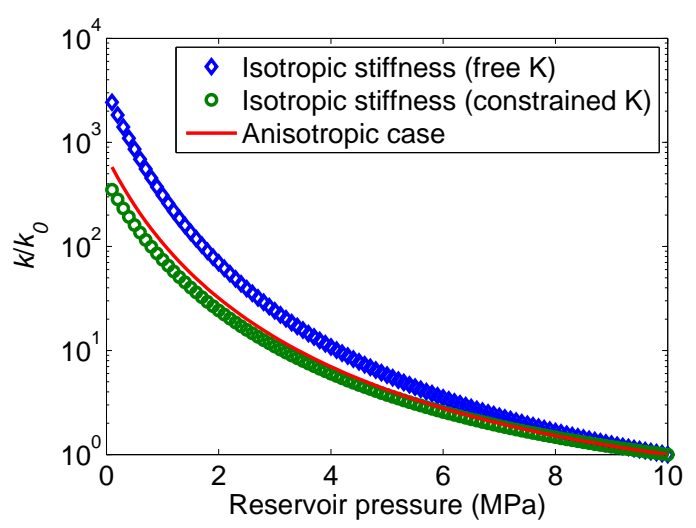

(b)

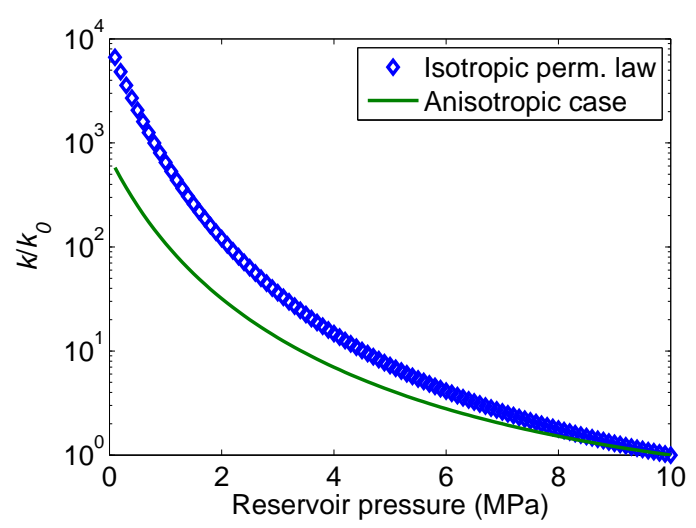

(d)

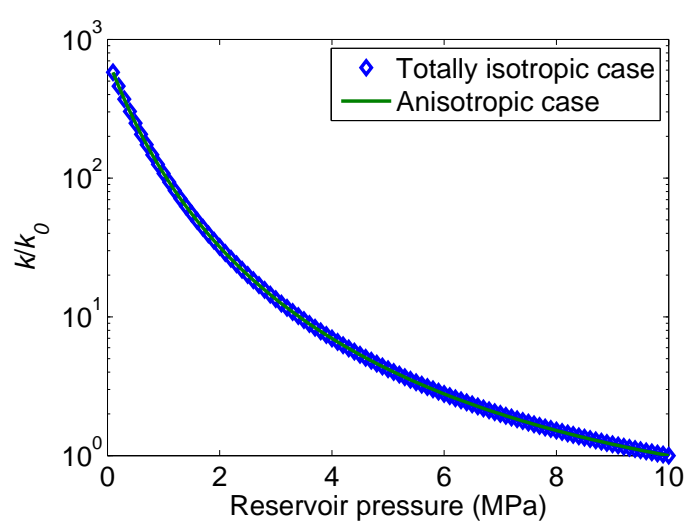

(f)

Figure 5: The $k-P$ relationships in oedometric (left) and isochoric (right) conditions for the equivalent materials when disregarding the anisotropy of stiffness (a)(b), the anisotropy of permeability dependence on stresses (c)(d), and the anisotropy of both $(\mathrm{e})(\mathrm{f})$. 


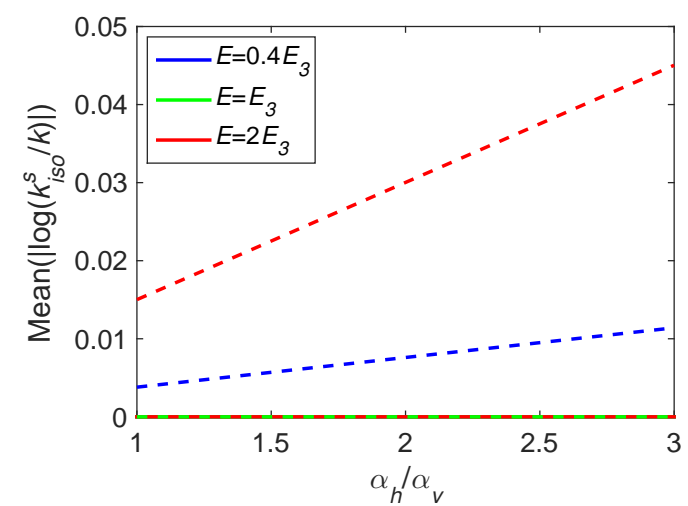

(a)

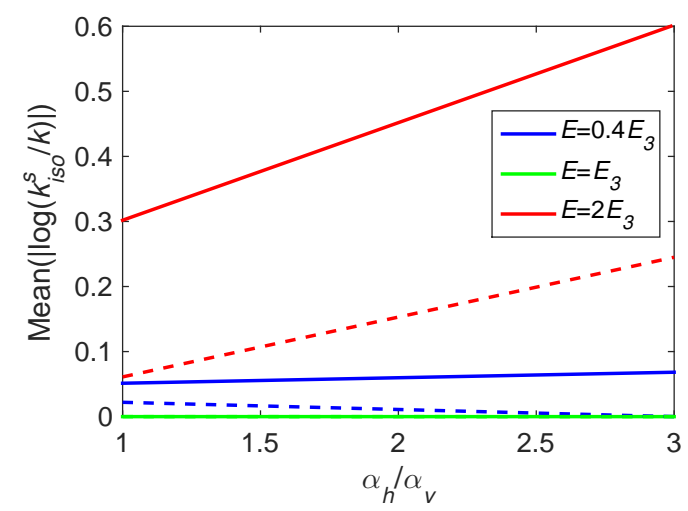

(c)

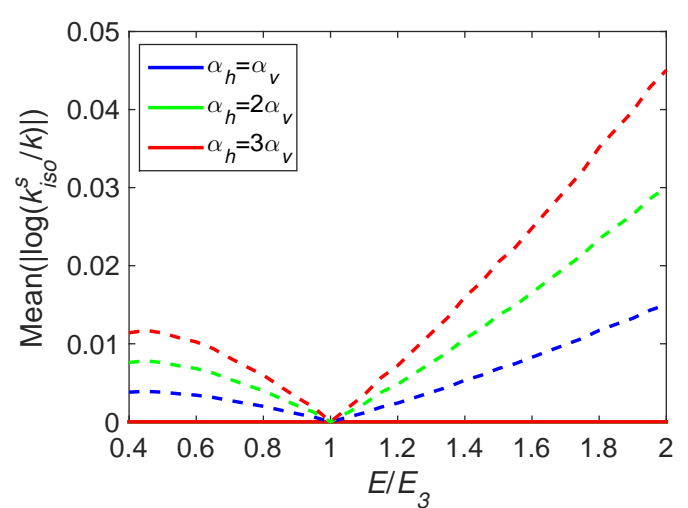

(b)

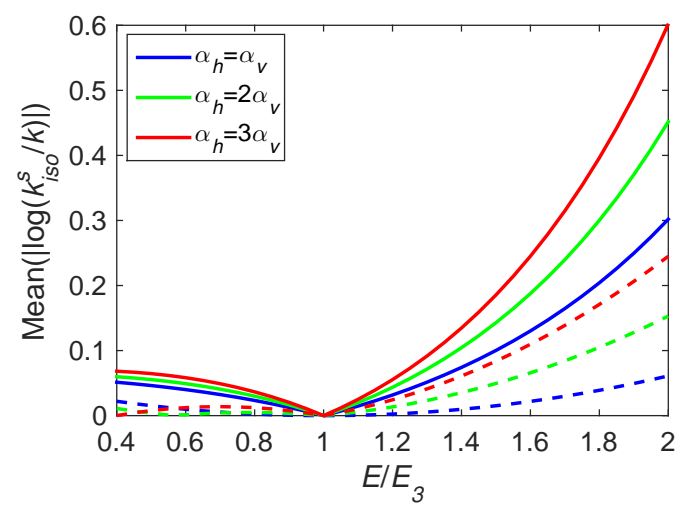

(d)

Figure 7: The errors on permeability introduced when disregarding the anisotropy of stiffness, depending on $\alpha_{h} / \alpha_{v}$ (left) and $E / E_{3}$ (right) in oedometric (top) and isochoric (bottom) conditions (solid line: free $K$ method, dashed line: constrained $K$ method). 


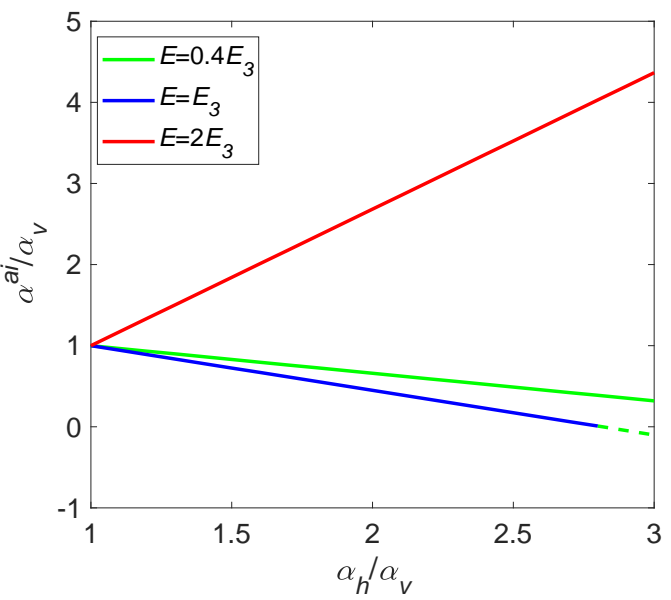

(a)

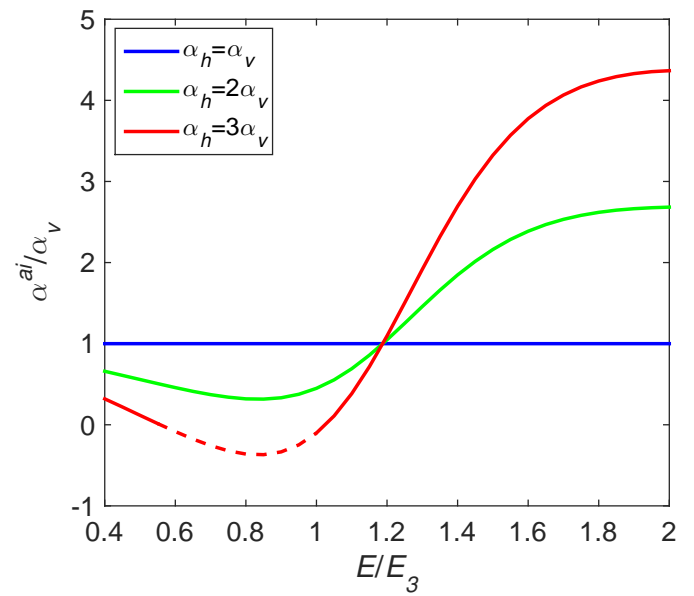

(b)

Figure 8: The assessed $\alpha^{a i}$ values in the case of neglecting the anisotropy of permeability dependence on stresses, depending on (a) $\alpha_{h} / \alpha_{v}$ and (b) $E / E_{3}$. The fittings provide sometimes negative $\alpha^{a i}$ values which are illustrated in dashed lines.

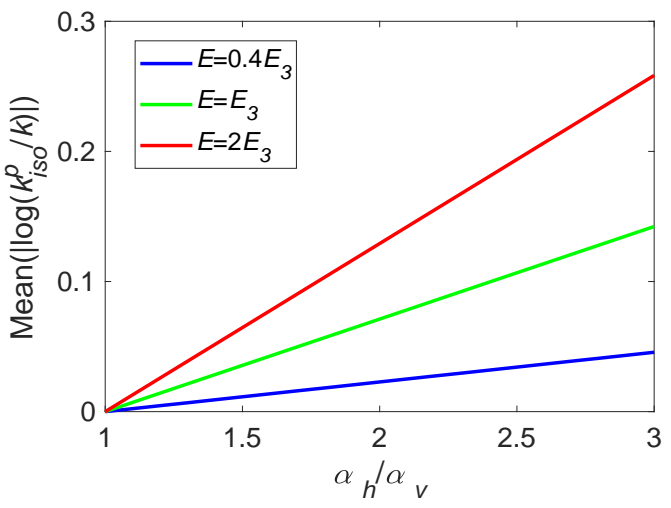

(a)

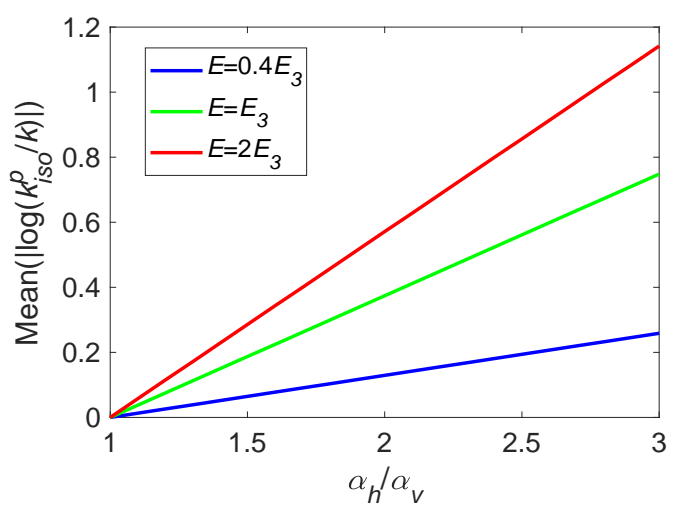

(c)

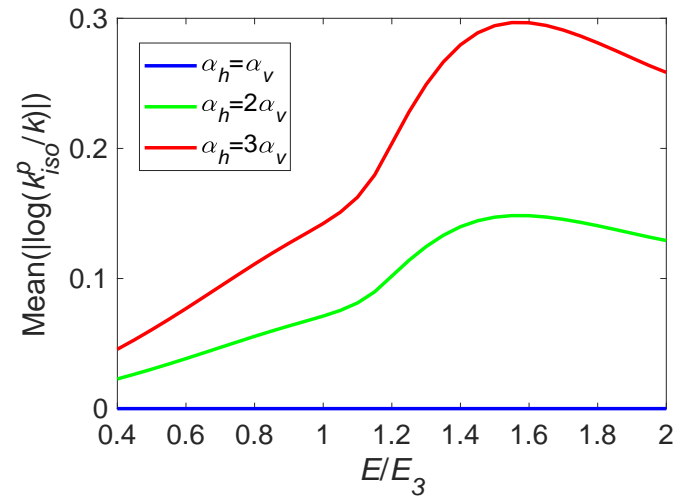

(b)

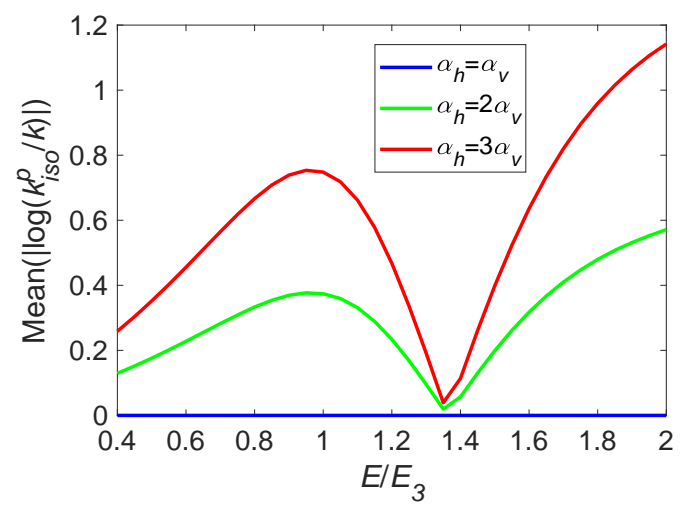

(d)

Figure 9: The errors on permeability introduced when disregarding the anisotropy of permeability dependence on stresses, depending on $\alpha_{h} / \alpha_{v}$ (left) and $E / E_{3}$ (right) in oedometric (top) and isochoric (bottom) conditions. 


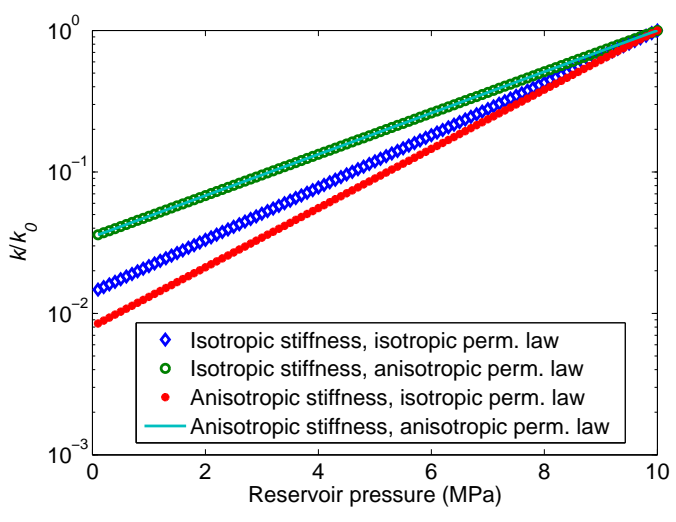

(a)

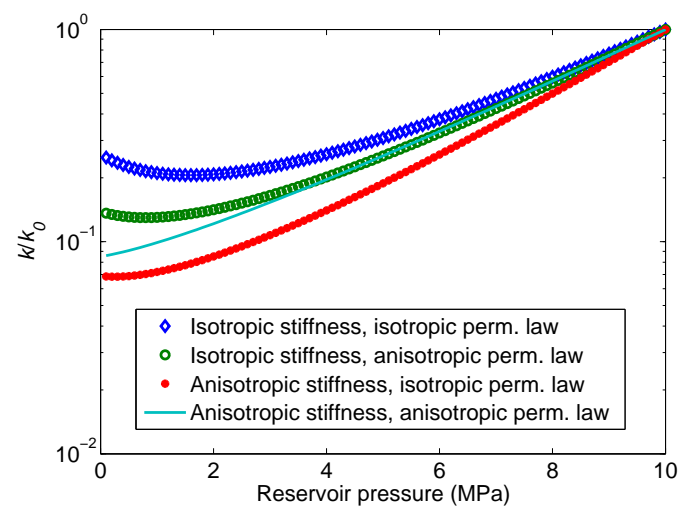

(b)

Figure 11: The $k-P$ curves for the totally isotropic case in (a) free swelling (i.e., $\Delta \sigma_{r}=\Delta \sigma_{a}=0$ ) and (b) inverse oedometric (i.e., $\Delta \sigma_{r}=0, \Delta \varepsilon_{a}=0$ ) conditions.

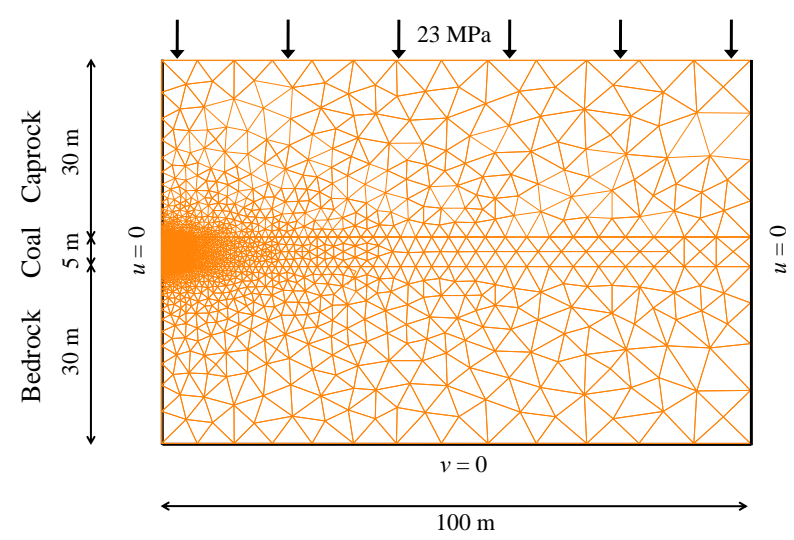

Figure 12: The model used for the reservoir simulation of methane recovery. 


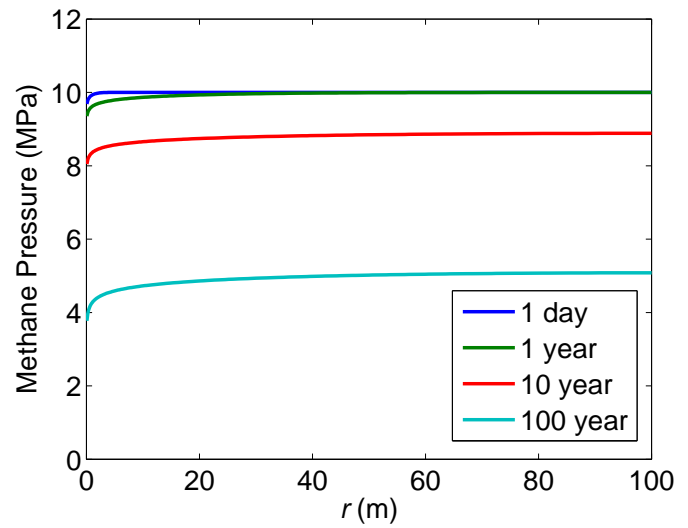

(a)

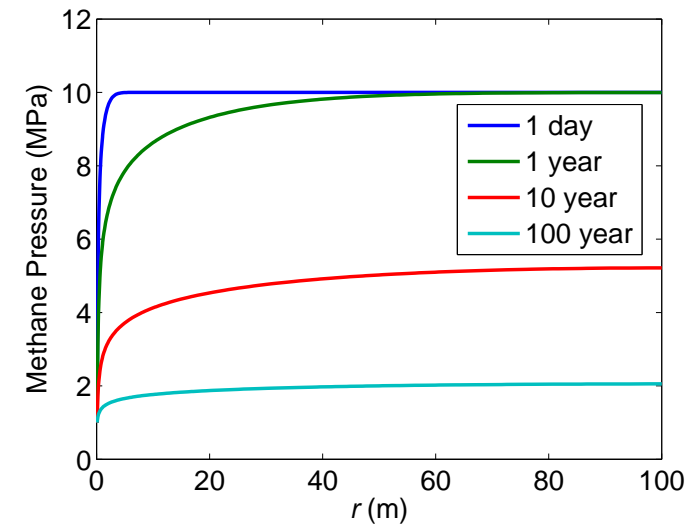

(b)

Figure 13: Methane pressure profiles at different times in the cases of constant flow (a) and of constant pressure (b) at wellbore from the reservoir simulation.

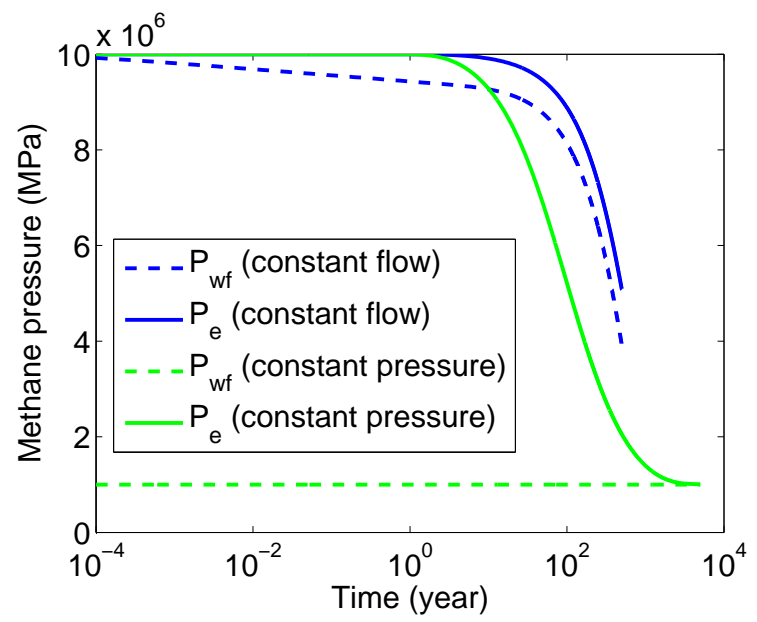

Figure 14: Evolution of borehole pressure $\left(P_{w f}\right)$ and pressure at the outer boundary $\left(P_{e}\right)$. 


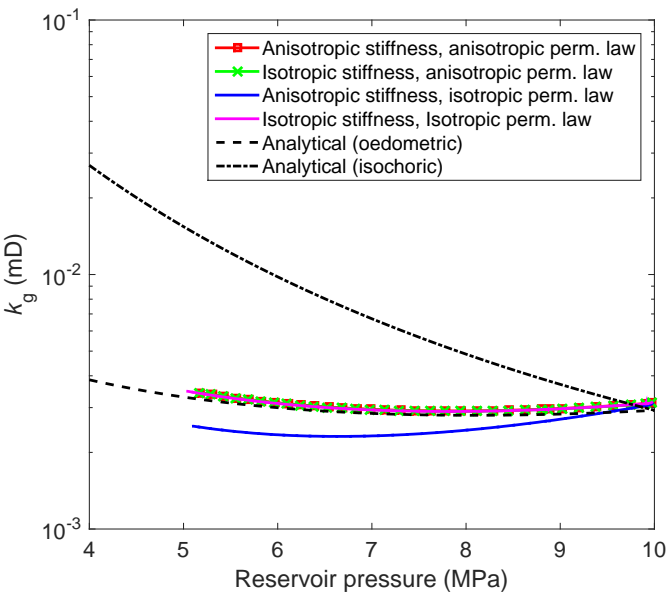

(a)

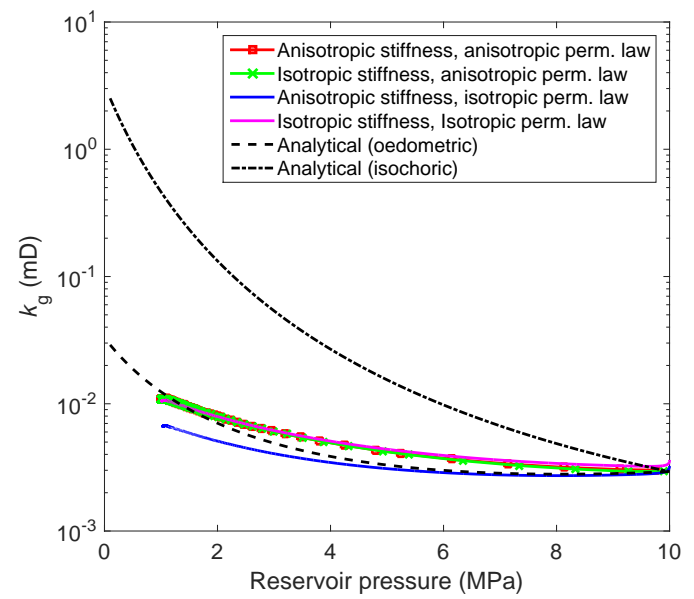

(b)

Figure 15: The $k_{g}-P$ curves evaluated from the reservoir simulation in the cases of constant flow (a) and of constant pressure (b) at the wellbore. 